\section{Características epidemiológicas da hanseníase nos idosos e comparação com outros grupos etários, Brasil (2016-2018)}

\author{
Epidemiological characteristics of leprosy in \\ elderly Brazilians and comparison with \\ other age groups (2016-2018)
}

\section{Características epidemiológicas de la hanseniasis en ancianos y comparación con otros grupos etarios, Brasil (2016-2018)}

\section{Resumo}

O objetivo foi descrever indicadores epidemiológicos e características dos casos novos de hanseníase em idosos no Brasil, no triênio 2016-2018, comparando a outros grupos etários. Estudo descritivo de corte transversal com dados do Sistema de Informação de Agravos de Notificação (SINAN). Os casos novos de hanseníase foram categorizados por grupos etários: 60 ou mais, 40-59, 15-39 e menores de 15 anos. Utilizou-se o teste qui-quadrado de Pearson para testar diferenças entre grupos. Foram notificados 81.205 casos novos de hanseníase no Brasil. Desses, 24,1\% foram em idosos, 37,7\% de 40-59 anos, 31,9\% de 15-39 e 6,3\% em menores de 15 anos. Nos idosos, foram observadas proporções maiores $(p<0,001)$ de casos no sexo masculino (60,1\%), com classificação operacional multibacilar (81,3\%) e com grau 2 de incapacidade física (GIF2) $(11,4 \%)$ em relação aos outros grupos. Contudo, a proporção de casos novos detectados em idosos, por exame de contatos (4,9\%), foi a menor entre todas as faixas etárias $(p<0,001)$. As taxas médias de detecção e de casos novos com GIF2 no diagnóstico foram maiores entre idosos (25,1/100 mil e 28,6/1 milhão de habitantes, respectivamente) em comparação aos demais grupos etários, para o país, regiões e Unidades da Federação. Foram observadas importantes diferenças nos perfis epidemiológico e clínico da hanseníase nos idosos, em relação às demais faixas etárias, destacando-se maiores proporções de casos multibacilares, de casos novos com GIF2 e baixa detecção por exame de contatos. Evidencia-se a necessidade do controle da hanseníase nessa população, visando a contribuir para a interrupção da transmissão da doença.

Hanseniase; Idoso; Epidemiologia Descritiva; Grupos Etários

\author{
Margarida Cristiana Napoleão Rocha 1 \\ Maurício Lisboa Nobre 2 \\ Leila Posenato Garcia 3
}

doi: 10.1590/0102/311X00048019

\author{
Correspondência \\ M. C. N. Rocha \\ Programa de Pós-graduação em Saúde Coletiva, Faculdade de \\ Ciências de Saúde, Universidade de Brasília. \\ Asa Norte, Brasília, DF 70910-900, Brasil. \\ marcrisrocha@gmail.com \\ 1 Faculdade de Ciências de Saúde, Universidade de Brasília, \\ Brasília, Brasil. \\ 2 Hospital Giselda Trigueiro, Secretaria Estadual de Saúde do \\ Rio Grande do Norte, Natal, Brasil. \\ 3 Instituto de Pesquisa Econômica Aplicada, Brasília, Brasil.
}




\section{Introdução}

O Brasil é o segundo país no mundo em número de casos novos de hanseníase 1. Em 2017, 26.686 casos novos foram notificados no país, dos quais $24,7 \%$ na população com 60 anos e mais de idade, 37,2\% de 40-59, 31,7\% de 15-39 e 6,4\% em menores de 15 anos (Departamento de Informática do SUS. http://tabnet.datasus.gov.br/cgi/sinannet/hanseniase/hans_indicadores.htm, acessado em 19/ Fev/2019).

A análise de indicadores de hanseníase por grupos etários é recomendada pela Estratégia Global de Hanseníase (2016-2020) com o intuito de caracterizar variações no tempo e no espaço. A estratificação desses indicadores por idade pode ser utilizada também como estimativa de risco de exposição ao bacilo e de detecção de casos da doença, bem como para subsidiar intervenções operacionais de acordo com a situação encontrada 2 . O aumento relativo de casos novos na população com 60 anos e mais de idade está associado à diminuição da transmissão da doença. No grupo menor de 15 anos significa persistência de transmissão ativa, já nos grupos de 15-39 e de 40-59 anos indica o acometimento de pessoas em idade economicamente ativa 2.

Ressalta-se a importância de que o indicador de detecção por grupos etários seja interpretado em conjunto com outros relacionados à qualidade da busca de casos, tais como as proporções de casos com grau 2 de incapacidade física (GIF2) e de casos em crianças, dentre os casos novos detectados e a taxa de GIF2 por hanseníase, calculada com base na população geral 3.

Nesse contexto, destaca-se que a população brasileira se encontra em processo de transição da estrutura etária, em que as mudanças mais notáveis ocorrerão nas faixas de idades extremas. Os menores de 15 anos perderão representatividade, já a proporção dos maiores de 60 anos será crescente ${ }^{3}$. O Censo Demográfico (2010) registrou 22.590 .599 idosos, 11,8\% da população. Estimativas do Instituto Brasileiro de Geografia e Estatística (IBGE), para 2050, preveem que esse contingente aumente para 66.457.570, o que corresponderá a 29,3\% da população (Departamento de Informática do SUS. http://tabnet.datasus.gov.br/cgi/sinannet/hanseniase/hans_indicadores.htm, acessado em 19/Fev/2019) e ultrapassará o número de menores de 15 anos 3.

A taxa média de detecção de casos novos de hanseníase no país, de 2014 a 2016, foi crescente conforme o aumento da faixa etária. Na população idosa foi cerca de oito vezes maior do que entre menores de 15 anos 4. De 2001 a 2013, houve redução progressiva da taxa de detecção de casos novos, acompanhada de aumento de $6 \%$ no percentual relativo de casos novos em idosos 5 . A chance de uma pessoa com 60 anos e mais de idade ter hanseníase foi maior quando comparada aos mais jovens $(\mathrm{OR}=2,64$; IC95\%: 1,$99 ; 3,52)$.

Apesar disso, os idosos acometidos pela doença são frequentemente excluídos das Estratégias Globais de Saúde. As estatísticas da Organização Mundial da Saúde (OMS) não analisam separadamente os indicadores da hanseníase nessa população 6 .

Estudos sobre hanseníase em idosos podem favorecer a tomada de decisão quanto à importância deste grupo etário na transmissão e nas incapacidades físicas. Além disso, propiciariam o entendimento das complicações, comorbidades e interações medicamentosas em decorrência da doença nos idosos 7 . Assim, conhecer o padrão da hanseníase nessa população pode subsidiar o planejamento das ações de vigilância e controle da doença.

O presente estudo tem como objetivo descrever indicadores epidemiológicos e características dos casos novos de hanseníase em idosos no Brasil, no triênio 2016-2018, comparando-os a outros grupos etários.

\section{Métodos}

\section{Desenho do estudo}

Foi realizado um estudo descritivo de corte transversal dos casos novos de hanseníase em residentes no Brasil, registrados no Sistema de Informação de Agravos de Notificação (SINAN), no período de 2016 a 2018, por faixas etárias. 


\section{Fonte de dados}

Foram usados dados secundários do SINAN, da Secretaria de Vigilância em Saúde, Ministério da Saúde, e a estimativa populacional para o ano de 2017 produzida pelo IBGE ("projeção da população das Unidades da Federação por sexo e grupos de idade: 2000-2030”) e disponibilizada pelo Departamento de Informática do Sistema Único de Saúde (DATASUS. http://www2.datasus.gov.br/DATA SUS/index.php?area=0206\&id=6942).

\section{População de estudo}

Foram incluídos todos os casos novos de hanseníase diagnosticados no Brasil e registrados no SINAN no período de 2016-2018.

\section{Variáveis de estudo}

A variável idade foi categorizada em quatro faixas etárias: 60 anos e mais, de 40-59, de 15-39 anos e em menores de 15 anos. Foram considerados idosos aqueles indivíduos com 60 ou mais anos de idade, segundo definição da OMS para países em desenvolvimento e do Brasil, de acordo com a Política Nacional de Saúde da Pessoa Idosa. Foram excluídos os casos novos com tipo de saída por erro diagnóstico.

Calculou-se também a proporção dos casos novos por faixa etária, segundo as seguintes variáveis: sexo, raça/cor da pele, escolaridade, classificação operacional, forma clínica, grau de incapacidade física no diagnóstico dentre os casos avaliados, modo de detecção do caso novo. Para o cálculo da proporção foi considerado no numerador o subconjunto desses casos segundo as variáveis epidemiológicas e clínicas, e no denominador o total de casos novos por faixa etária.

Para a raça/cor da pele, considerou-se a definição dada pelo Estatuto da Igualdade Racial 8. Para a escolaridade foram adaptadas as categorias do SINAN aos parâmetros do nível de instrução do IBGE 9.

\section{Indicadores}

Para as análises dos indicadores epidemiológicos foram calculadas a taxa média de detecção de casos novos de hanseníase, por 100 mil habitantes, e a taxa média de casos novos com GIF2 no momento do diagnóstico, por 1 milhão de habitantes ${ }^{10}$. No numerador da taxa média de detecção, utilizou-se a média do número de casos novos em idosos, registrados no período de 2016-2018, e no denominador empregou-se a população brasileira estimada pelo IBGE para o ano de 2017, o ano central do período analisado. Para a taxa de casos novos com GIF2 no diagnóstico usou-se a média do número de casos novos em idosos com GIF2, registrados no período de 2016-2018, e no denominador a população brasileira estimada pelo IBGE para o ano de 2017, o ano central do período analisado. Os indicadores foram calculados para o Brasil, suas cinco regiões (Norte, Nordeste, Sudeste, Sul e Centro-oeste) e as 27 Unidades da Federação (UF).

Foram calculadas as razões entre as taxas de detecção geral e de GIF2 nos idosos em relação aos grupos de 40-59 anos, de 15-39 e menores de 15 anos. Devido à ausência de notificação de casos com GIF2 em menores de 15 anos em algumas UF, as razões foram calculadas para Brasil e regiões.

\section{Gerenciamento e análise de dados}

As análises foram realizadas com o auxílio do software Stata versão 15.0 (https://www.stata.com). Usou-se o teste qui-quadrado de Pearson para verificar as diferenças entre as características da doença nos idosos em relação aos demais grupos etários. Foi adotado o nível de 1\% de significância. 


\section{Considerações éticas}

O presente estudo foi aprovado pelo Comitê de Ética da Faculdade de Ciências da Saúde da Universidade de Brasília, com o número do CAAE 77799417.6.0000.0030 e parecer número 2.411.253, emitido em 2 de dezembro de 2017.

\section{Resultados}

No Brasil, de 2016-2018, foram diagnosticados 81.205 casos novos de hanseníase. Desses, 19.582 $(24,1 \%)$ ocorreram em pessoas com 60 anos e mais de idade, $30.593(37,7 \%)$ de 40-59, $25.896(31,9 \%)$ de 15 -39 e 5.134 (6,3\%) em menores de 15 anos (Tabela 1).

Em todos os grupos etários observou-se o predomínio do sexo masculino entre os casos novos de hanseníase, com maior proporção entre idosos $(60,1 \%)(\mathrm{p}<0,001)$. A raça/cor da pele negra foi a mais frequente entre os casos de hanseníase em todas as faixas etárias; nos idosos esta proporção foi de 64,8\%, com diferença significativa em relação às demais faixas etárias ( $\mathrm{p}<0,001)$. Destaca-se ainda o elevado percentual de casos novos em idosos $(66,1 \%)$ sem escolaridade ou com o Ensino Fundamental incompleto (Tabela 1).

\section{Tabela 1}

Número absoluto e proporção de casos novos de hanseníase, segundo características epidemiológicas e clínicas, por faixa etária. Brasil, $2016-2018$.

\begin{tabular}{|c|c|c|c|c|c|c|c|c|}
\hline \multirow[t]{3}{*}{ Variáveis } & \multicolumn{8}{|c|}{ Faixa etária (anos) } \\
\hline & \multicolumn{2}{|c|}{$<15$} & \multicolumn{2}{|c|}{$15-39$} & \multicolumn{2}{|c|}{$40-59$} & \multicolumn{2}{|c|}{60 ou mais } \\
\hline & $\mathbf{n}$ & $\%$ & $\mathbf{n}$ & $\%$ & $\mathbf{n}$ & $\%$ & $\mathbf{n}$ & $\%$ \\
\hline Total & 5.134 & 100 & 25.896 & 100 & 30.593 & 100 & 19.582 & 100 \\
\hline \multicolumn{9}{|l|}{ Sexo } \\
\hline Masculino & 2.665 & 51,9 & 14.153 & 54,7 & 16.238 & 53,1 & 11.760 & 60,1 \\
\hline Feminino & 2.469 & 48,1 & 11.741 & 45,3 & 14.353 & 46,9 & 7.819 & 39,9 \\
\hline Valor de $p$ * & \multicolumn{2}{|c|}{$<0,001$} & \multicolumn{2}{|c|}{$<0,001$} & \multicolumn{2}{|c|}{$<0,001$} & \multicolumn{2}{|c|}{1,000} \\
\hline \multicolumn{9}{|l|}{ Raça/cor da pele } \\
\hline Branca & 795 & 15,5 & 5.292 & 20,4 & 7.470 & 24,4 & 5.955 & 30,4 \\
\hline Negra & 4.087 & 79,6 & 19.319 & 74,6 & 21.654 & 70,8 & 12.689 & 64,8 \\
\hline Amarela & 52 & 1,0 & 285 & 1,1 & 295 & 1,0 & 187 & 1,0 \\
\hline Indígena & 24 & 0,5 & 143 & 0,6 & 139 & 0,5 & 80 & 0,4 \\
\hline Valor de $p$ * & \multicolumn{2}{|c|}{$<0,001$} & \multicolumn{2}{|c|}{$<0,001$} & \multicolumn{2}{|c|}{$<0,001$} & \multicolumn{2}{|c|}{1,000} \\
\hline \multicolumn{9}{|l|}{ Escolaridade ** } \\
\hline Sem instrução e Ensino Fundamental incompleto & 3.516 & 68,5 & 8.507 & 32,9 & 16.429 & 53,7 & 12.944 & 66,1 \\
\hline Ensino Fundamental completo e Médio incompleto & 297 & 5,8 & 5.274 & 20,4 & 3.321 & 10,9 & 1.256 & 6,4 \\
\hline Ensino Médio completo e Superior incompleto & 12 & 0,2 & 6.747 & 26,1 & 4.019 & 13,1 & 1.073 & 5,5 \\
\hline Ensino Superior completo & 1 & 0,0 & 1.144 & 4,4 & 1.274 & 4,2 & 382 & 2,0 \\
\hline \multicolumn{9}{|l|}{ Classificação operacional } \\
\hline Paucibacilar & 2.302 & 44,8 & 7.372 & 28,5 & 7.196 & 23,5 & 3.666 & 18,7 \\
\hline Multibacilar & 2.830 & 55,1 & 18.521 & 71,5 & 23.396 & 76,5 & 15.914 & 81,3 \\
\hline Valor de $p$ * & \multicolumn{2}{|c|}{$<0,001$} & \multicolumn{2}{|c|}{$<0,001$} & \multicolumn{2}{|c|}{$<0,001$} & \multicolumn{2}{|c|}{1,000} \\
\hline \multicolumn{9}{|l|}{ Forma clínica } \\
\hline Indeterminada & 1.203 & 23,4 & 4.024 & 15,5 & 3.449 & 11,3 & 1.613 & 8,2 \\
\hline Tuberculoide & 1.050 & 20,5 & 3.404 & 13,1 & 4.076 & 13,3 & 2.423 & 12,4 \\
\hline Dimorfa & 2.196 & 42,8 & 12.314 & 47,6 & 15.403 & 50,3 & 9.931 & 50,7 \\
\hline Virchowiana & 322 & 6,3 & 3.853 & 14,9 & 4.899 & 16,0 & 3.749 & 19,1 \\
\hline Valor de $p$ * & \multicolumn{2}{|c|}{$<0,001$} & \multicolumn{2}{|c|}{$<0,001$} & \multicolumn{2}{|c|}{$<0,001$} & \multicolumn{2}{|c|}{1,000} \\
\hline
\end{tabular}

(continua) 
Tabela 1 (continuação)

\begin{tabular}{|c|c|c|c|c|c|c|c|c|}
\hline \multirow[t]{3}{*}{ Variáveis } & \multicolumn{8}{|c|}{ Faixa etária (anos) } \\
\hline & \multicolumn{2}{|c|}{$<15$} & \multicolumn{2}{|c|}{$15-39$} & \multicolumn{2}{|c|}{$40-59$} & \multicolumn{2}{|c|}{60 ou mais } \\
\hline & $\mathbf{n}$ & $\%$ & $\mathbf{n}$ & $\%$ & $\mathbf{n}$ & $\%$ & $\mathbf{n}$ & $\%$ \\
\hline \multicolumn{9}{|l|}{ Grau de incapacidade * } \\
\hline Grau 0 & 3.886 & 75,7 & 16.292 & 62,9 & 16.832 & 55,0 & 9.047 & 46,2 \\
\hline Grau 1 & 604 & 11,8 & 5.146 & 19,9 & 7.790 & 25,5 & 5.697 & 29,1 \\
\hline Grau 2 & 127 & 2,5 & 1.389 & 5,4 & 2.185 & 7,1 & 2.228 & 11,4 \\
\hline Valor de p * & \multicolumn{2}{|c|}{$<0,001$} & \multicolumn{2}{|c|}{$<0,001$} & \multicolumn{2}{|c|}{$<0,001$} & \multicolumn{2}{|c|}{1,000} \\
\hline \multicolumn{9}{|c|}{ Modo de detecção do caso novo } \\
\hline Encaminhamento & 1.917 & 37,3 & 10.804 & 41,7 & 14.050 & 45,9 & 10.025 & 51,2 \\
\hline Demanda espontânea & 1.591 & 31,0 & 10.388 & 40,1 & 12.291 & 40,2 & 7.230 & 36,9 \\
\hline Exame de coletividade & 482 & 9,4 & 1.085 & 4,2 & 1.177 & 3,8 & 669 & 3,4 \\
\hline Exame de contatos & 1.021 & 19,9 & 2.885 & 11,1 & 2.187 & 7,1 & 955 & 4,9 \\
\hline Outros modos & 66 & 1,3 & 439 & 1,7 & 543 & 1,8 & 495 & 2,5 \\
\hline Valor de $p$ * & \multicolumn{2}{|c|}{$<0,001$} & \multicolumn{2}{|c|}{$<0,001$} & \multicolumn{2}{|c|}{$<0,001$} & \multicolumn{2}{|c|}{1,000} \\
\hline
\end{tabular}

Fonte: Sistema de Informação de Agravos de Notificação.

Nota: ignorados (excluídos do teste qui-quadrado): sexo (< 15 anos: 0; 15-39 anos: 2; 40-59 anos: 2; 60 anos e mais: 3); raça/cor da pele (< 15 anos: 176; 15-39 anos: 857; 40-59 anos: 1.035; 60 anos e mais: 671); escolaridade (< 15 anos: 1.308; 15-39 anos: 4.224; 40-59 anos: 5.550; 60 anos e mais: 3.927); classificação operacional (< 15 anos: 2; 15-39 anos: 3; 40-59 anos: 1; 60 anos e mais: 2); forma clínica (< 15 anos: 363 ; $15-39$ anos: 2.301; 40-59 anos: 2.766; 60 anos e mais: 1.866); grau de incapacidade (< 15 anos: 517; 15-39 anos: 3.069; 40-59 anos: $3.786 ; 60$ anos e mais: 2.610); modo de detecção do caso novo (< 15 anos: 57; 15-39 anos: 295; 40-59 anos: 345; 60 anos e mais: 208); contatos examinados (<15 anos: 378; 15-39 anos: 2.724; 40-59 anos: 3.676; 60 anos e mais: 2.692).

* Valor de $p$ do teste qui-quadrado de Pearson.

** Não realizado o teste, pois se espera que a distribuição da escolaridade entre as faixas etárias será diferente.

A proporção de casos novos com classificação operacional multibacilar foi ascendente com o aumento da faixa etária, atingindo $81,3 \%$ dos idosos, concomitante ao aumento na proporção das formas dimorfa e virchowiana, que corresponderam a 50,7\% e 19,1\%, respectivamente, dos casos em idosos ( $\mathrm{p}<0,001)$. A proporção de casos com GIF2 foi maior entre os idosos do que nos demais grupos (11,4\%), cerca de 1,6 vez superior ao valor do grupo etário de 40-59 anos (7,1\%), 2 vezes ao grupo de $15-39(5,4 \%)$ e 5 vezes ao de menores de 15 anos (2,5\%) ( $<<0,001)$ (Tabela 1$)$.

O modo de detecção dos casos novos mais frequentes em todas as faixas etárias foi o encaminhamento, maior nos idosos $(51,2 \%)$ ( $\mathrm{p}<0,001)$. Em contrapartida, a detecção por exame de contatos foi menor nesse grupo etário (4,9\%), aproximadamente 2 vezes menos frequente em relação às pessoas de 15-39 anos (11,1\%) e quatro vezes $(19,9 \%)$ às menores de 15 anos $(\mathrm{p}<0,001)$ (Tabela 1).

A taxa média de detecção de hanseníase, por 100 mil habitantes, foi crescente entre os grupos etários e maior nos idosos do que nas demais faixas etárias, tanto para o Brasil (25,1 casos novos por 100 mil habitantes/ano) como para todas as regiões. A Região Norte apresentou a maior taxa em idosos $(73,7)$, seguida da Centro-oeste $(71,6)$ e Nordeste $(47,8)$, já as regiões Sudeste $(8,9)$ e Sul $(6,2)$ registraram os menores valores (Tabela 2 ).

No Brasil e em todas as regiões as razões entre taxas calculadas foram superiores a um. Para o país, a razão da taxa de detecção nos idosos foi de 1,2; 2,5 e 6,8 vezes superior ao grupo de 40-59 anos, de 15-39 e de menores de 15 anos, respectivamente (Figura 1).

Valores superiores à média nacional foram observados em algumas regiões. A razão entre as taxas dos idosos e os menores de 15 anos foi de 30,4 e 10,1 vezes maior nas regiões Sul e Sudeste, respectivamente. Para as pessoas com 60 anos e mais em relação às de 15-39 anos, foi 3,8 e 3,2 vezes superior nas regiões Sul e Nordeste, respectivamente, já entre o grupo etário de 40 a 59 anos foi de 1,5 vez maior na Nordeste e 1,4 vez superior nas regiões Norte, Sudeste e Sul (Figura 1). 


\section{Tabela 2}

Taxa média de detecção de casos novos de hanseníase (por 100 mil habitantes/ano), segundo faixas etárias. Brasil, regiões e Unidades da Federação (UF), 2016-2018.

\begin{tabular}{|c|c|c|c|c|c|}
\hline \multirow[t]{2}{*}{ Regiões/UFs } & \multicolumn{4}{|c|}{ Faixa etária (anos) } & \multirow[t]{2}{*}{ Total } \\
\hline & $<15$ & $15-39$ & $40-59$ & 60 e mais & \\
\hline Norte & 8,8 & 25,6 & 52,1 & 73,7 & 29,6 \\
\hline Rondônia & 5,8 & 27,6 & 56,7 & 64,3 & 32,0 \\
\hline Acre & 4,2 & 17,2 & 24,7 & 28,5 & 15,2 \\
\hline Amazonas & 3,6 & 10,3 & 19,0 & 24,6 & 10,8 \\
\hline Roraima & 7,2 & 17,2 & 36,1 & 58,5 & 20,2 \\
\hline Pará & 10,7 & 27,4 & 49,2 & 72,2 & 30,5 \\
\hline Amapá & 2,7 & 12,7 & 22,4 & 31,6 & 12,5 \\
\hline Tocantins & 24,3 & 68,8 & 169,6 & 218,8 & 91,6 \\
\hline Nordeste & 5,9 & 15,1 & 32,8 & 47,8 & 20,2 \\
\hline Maranhão & 15,3 & 39,1 & 77,0 & 123,3 & 46,4 \\
\hline Piauí & 7,7 & 21,8 & 53,0 & 79,2 & 31,3 \\
\hline Ceará & 3,4 & 10,4 & 32,6 & 52,3 & 18,2 \\
\hline Rio Grande do Norte & 1,0 & 4,0 & 11,8 & 17,7 & 6,6 \\
\hline Paraíba & 2,6 & 9,1 & 19,4 & 26,8 & 11,9 \\
\hline Pernambuco & 7,7 & 17,6 & 37,1 & 46,2 & 22,9 \\
\hline Alagoas & 2,8 & 7,0 & 16,4 & 21,8 & 9,3 \\
\hline Sergipe & 4,2 & 10,2 & 23,8 & 39,2 & 14,5 \\
\hline Bahia & 3,6 & 10,4 & 22,7 & 31,8 & 14,0 \\
\hline Sudeste & 0,9 & 3,0 & 6,4 & 8,9 & 4,3 \\
\hline Minas Gerais & 1,3 & 3,3 & 8,3 & 10,8 & 5,2 \\
\hline Espírito Santo & 2,7 & 10,0 & 16,6 & 21,4 & 11,5 \\
\hline Rio de Janeiro & 1,3 & 3,9 & 7,9 & 11,0 & 5,6 \\
\hline São Paulo & 0,3 & 2,0 & 4,1 & 6,2 & 2,8 \\
\hline Sul & 0,2 & 1,6 & 4,3 & 6,2 & 2,7 \\
\hline Paraná & 0,2 & 2,7 & 8,2 & 12,9 & 5,0 \\
\hline Santa Catarina & 0,2 & 1,5 & 3,0 & 3,1 & 1,9 \\
\hline Rio Grande do Sul & 0,2 & 0,5 & 1,4 & 2,3 & 1,0 \\
\hline Centro-oeste & 7,1 & 25,0 & 63,7 & 71,6 & 35,3 \\
\hline Mato Grosso do Sul & 1,9 & 9,0 & 25,2 & 34,5 & 14,1 \\
\hline Mato Grosso & 22,6 & 78,9 & 202,7 & 217,5 & 108,6 \\
\hline Goiás & 3,5 & 14,5 & 36,3 & 48,2 & 21,1 \\
\hline Distrito Federal & 0,9 & 4,5 & 8,2 & 11,2 & 5,3 \\
\hline Brasil & 3,7 & 10,1 & 20,3 & 25,1 & 13,0 \\
\hline
\end{tabular}

Fonte: Departamento de Informática do Sistema Único de Saúde. Sistema de Informação de Agravos de Notificação. http://tabnet.datasus.gov.br/cgi/sinannet/hanseniase/hans_indicadores.htm (acessado em 19/Fev/2019).

As maiores taxas de detecção de casos novos por 100 mil habitantes em pessoas com 60 ou mais anos de idade foram observadas em Tocantins (218,8), Mato Grosso (217,5), Maranhão (123,3), Piauí $(79,2)$ e Pará $(72,2)$. As menores taxas de detecção em idosos ocorreram nos estados do Rio Grande do Sul $(2,3)$, Santa Catarina (3,1) e São Paulo $(6,2)$ (Tabela 2).

A taxa média de casos novos com GIF2 no diagnóstico por 1 milhão de habitantes também foi maior nos idosos, tanto para o Brasil (28,6 casos novos com GIF2 por 1 milhão habitantes/ano) como para todas as regiões. A Região Norte apresentou a maior taxa de incapacidade em idosos $(98,2)$, seguida da Centro-oeste $(67,9)$ e Nordeste $(43,6)$, já a Sudeste $(14,7)$ e a Sul $(10,2)$ apresentaram taxas bem menores (Tabela 3). 
Figura 1

Razão entre taxas de detecção de casos novos de hanseníase, segundo faixas etárias. Brasil e regiões, 2016-2018.

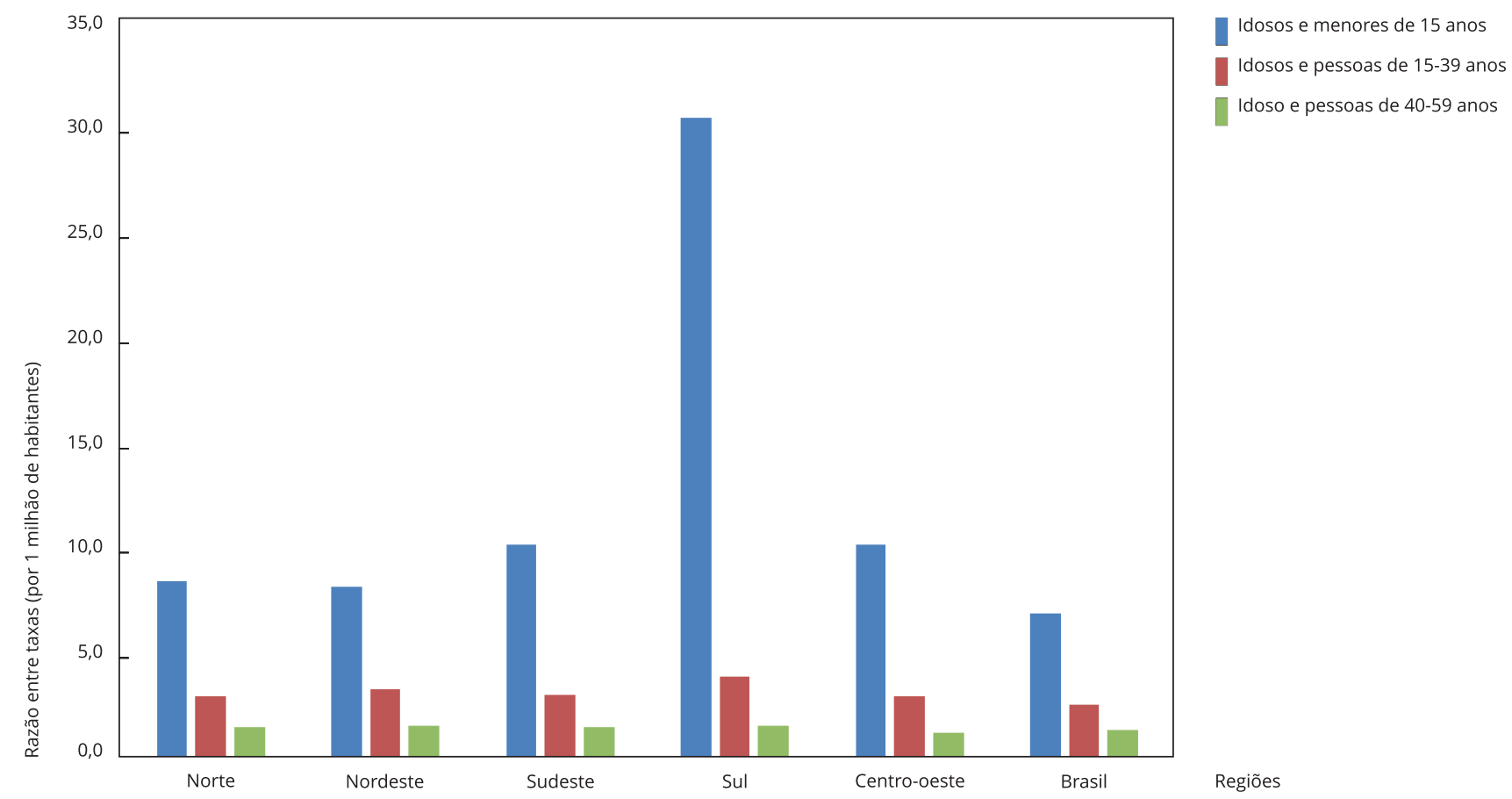

As razões das taxas de GIF2 no Brasil foram de 2,0; 5,3 e 31,2 vezes superiores nos idosos em relação aos grupos de 40-59 anos, de 15-39 e em menores de 15 anos, respectivamente. Entre as taxas dos idosos e o grupo etário de 40-59 anos foi cerca de 2 vezes maior em todas as regiões. Para as pessoas com 60 anos e mais e as de 15-39 anos, a razão foi de 8,4 e 7,0 vezes superior nas regiões Centro-oeste e Norte, respectivamente, e entre os menores de 15 anos foi de 109,2 e 45,1 vezes maior nas regiões Sudeste e Centro-oeste, respectivamente (Figura 2).

Em todas as UFs a taxa de GIF2 por 1 milhão de habitantes também foi maior nos idosos. Os estados do Tocantins $(302,1)$, Mato Grosso $(188,3)$ e Maranhão $(119,7)$ apresentaram os maiores valores. As menores taxas de GIF2 nos idosos foram observadas no Rio Grande do Sul (6,0), Santa Catarina $(6,9)$ e São Paulo $(11,6)$ (Tabela 3). 


\section{Tabela 3}

Taxa média de casos novos de hanseníase com grau 2 de incapacidade física no diagnóstico (por 1 milhão de habitantes/ ano). Brasil, regiões e Unidades da Federação (UF), 2016-2018.

\begin{tabular}{|c|c|c|c|c|c|}
\hline \multirow[t]{2}{*}{ Regiões/UFs } & \multicolumn{4}{|c|}{ Faixa etária (anos) } & \multirow[t]{2}{*}{ Total } \\
\hline & $<15$ & $15-39$ & $40-59$ & 60 e mais & \\
\hline Norte & 2,7 & 13,9 & 41,9 & 98,2 & 22,6 \\
\hline Rondônia & 1,5 & 19,1 & 38,0 & 69,4 & 23,3 \\
\hline Acre & 1,3 & 3,7 & 29,2 & 69,7 & 12,1 \\
\hline Amazonas & 0,5 & 6,8 & 25,0 & 46,4 & 10,9 \\
\hline Roraima & 2,1 & 14,1 & 20,6 & 73,1 & 15,3 \\
\hline Pará & 3,5 & 15,0 & 39,1 & 88,0 & 22,3 \\
\hline Amapá & 2,7 & 3,7 & 9,1 & 64,6 & 7,9 \\
\hline Tocantins & 7,3 & 32,0 & 128,5 & 302,1 & 70,1 \\
\hline Nordeste & 1,4 & 8,0 & 20,4 & 43,6 & 12,9 \\
\hline Maranhão & 4,8 & 20,3 & 46,2 & 119,7 & 29,1 \\
\hline Piauí & 1,2 & 10,3 & 31,5 & 70,6 & 19,2 \\
\hline Ceará & 1,5 & 7,2 & 20,9 & 54,5 & 14,1 \\
\hline Rio Grande do Norte & 0,4 & 2,0 & 8,3 & 19,4 & 5,0 \\
\hline Paraíba & 0,3 & 7,2 & 14,4 & 35,5 & 10,5 \\
\hline Pernambuco & 1,0 & 6,8 & 20,3 & 32,2 & 11,4 \\
\hline Alagoas & 0,4 & 6,1 & 13,1 & 17,3 & 7,1 \\
\hline Sergipe & 0,6 & 7,7 & 18,4 & 43,9 & 11,8 \\
\hline Bahia & 0,4 & 5,1 & 14,6 & 25,0 & 8,4 \\
\hline Sudeste & 0,1 & 2,4 & 7,0 & 14,7 & 4,9 \\
\hline Minas Gerais & 0,3 & 2,6 & 8,5 & 19,5 & 6,0 \\
\hline Espírito Santo & 0,0 & 3,8 & 12,0 & 17,2 & 6,7 \\
\hline Rio de Janeiro & 0,0 & 4,3 & 8,1 & 16,1 & 6,3 \\
\hline São Paulo & 0,1 & 1,6 & 5,4 & 11,6 & 3,7 \\
\hline Sul & 0,2 & 1,5 & 4,7 & 10,2 & 3,4 \\
\hline Paraná & 0,3 & 1,8 & 8,1 & 17,3 & 5,2 \\
\hline Santa Catarina & 0,2 & 2,0 & 3,5 & 6,9 & 2,7 \\
\hline Rio Grande do Sul & 0,2 & 0,8 & 2,0 & 6,0 & 1,9 \\
\hline Centro-oeste & 1,5 & 8,1 & 33,7 & 67,9 & 19,2 \\
\hline Mato Grosso do Sul & 0,0 & 3,9 & 25,1 & 46,8 & 12,9 \\
\hline Mato Grosso & 3,3 & 19,8 & 85,1 & 188,3 & 47,8 \\
\hline Goiás & 1,4 & 6,8 & 22,3 & 45,0 & 13,6 \\
\hline Distrito Federal & 1,1 & 2,4 & 10,9 & 17,2 & 5,7 \\
\hline Brasil & 0,9 & 5,4 & 14,5 & 28,6 & 9,5 \\
\hline
\end{tabular}

Fonte: Departamento de Informática do Sistema Único de Saúde. Sistema de Informação de Agravos de Notificação. http://tabnet.datasus.gov.br/cgi/sinannet/hanseniase/hans_indicadores.htm (acessado em 19/Fev/2019). 


\section{Figura 2}

Razão entre taxas de grau 2 de incapacidade física de hanseníase (por 1 milhão de habitantes), segundo faixas etárias. Brasil e regiões, $2016-2018$.

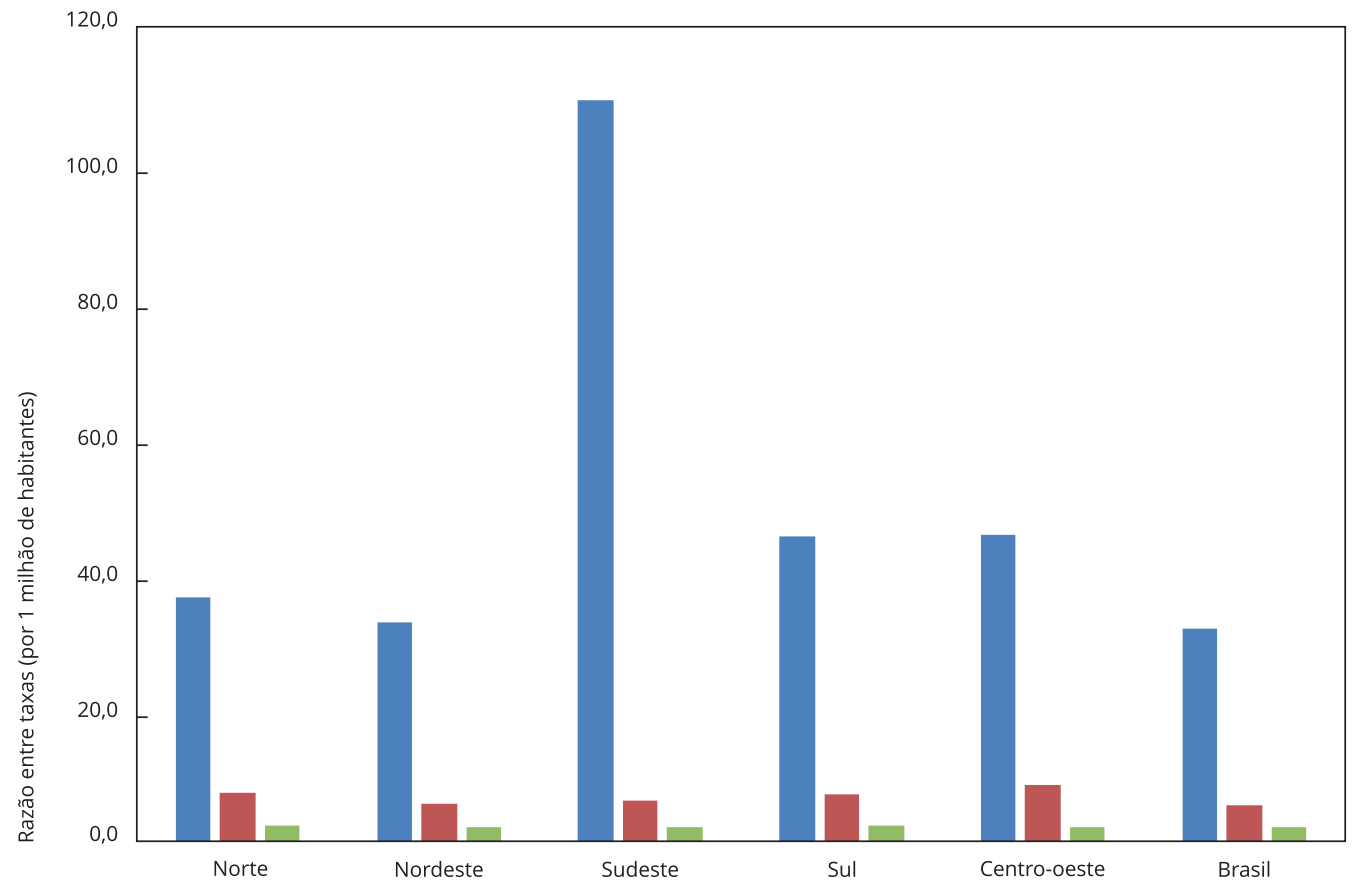

Idosos e menores de 15 anos

Idosos e pessoas de 15-39 anos

Idoso e pessoas de 40-59 anos

\section{Discussão}

O presente estudo revelou que entre os casos novos de hanseníase em idosos houve maiores proporções de homens, casos multibacilares e com GIF2. Além disso, as taxas de detecção de hanseníase e de casos novos com GIF2 foram mais elevadas nos idosos, quando comparadas às demais faixas etárias, no Brasil, regiões e UF. Esse contexto reforça que a busca de casos nesse grupo populacional pode acelerar a detecção, o tratamento e consequentemente o bloqueio das fontes de infecção da doença 5 .

O predomínio do sexo masculino entre os casos novos em todos os grupos etários, especialmente entre idosos, foi encontrado em outros estudos como os de Diniz \& Maciel 7 e Nogueira et al. ${ }^{11}$. No mesmo sentido, Nobre et al. 5 encontraram diferenças estatisticamente significativas nas taxas médias de detecção entre os sexos, mais elevadas no sexo masculino, sobretudo na faixa etária de 60 anos e mais.

O maior acometimento dos homens pode ser explicado pelo menor cuidado dispensado à saúde por parte deste grupo populacional ${ }^{12}$, mas também é necessário investigar aspectos relacionados à susceptibilidade genética e à possível interferência hormonal na resposta imune ao Microbacterium leprae 6. Independentemente das causas associadas a esse padrão epidemiológico, esses resultados indicam um desafio adicional para o controle da endemia.

Destacam-se as altas proporções de pessoas de cor da pele negra e sem instrução ou com ensino fundamental incompleto, entre os casos de hanseníase, em todas as faixas etárias, com 64,8\% e 66,1\% entre os idosos, respectivamente. Tais achados foram semelhantes àqueles de Silva et al. 13 e Diniz \& Maciel 8. Outros estudos revelaram associação entre altas taxas de detecção de hanseníase e fatores socioeconômicos precários, como baixa escolaridade e alto índice de vulnerabilidade social 14,15.

Neste estudo, a classificação operacional multibacilar foi mais frequente nos idosos, resultado semelhante ao encontrado em outras pesquisas 5,16. As formas multibacilares ocorrem pela instabili- 
dade imunológica contra o M. leprae em indivíduos que apresentam menor imunidade celular contra o bacilo. Pessoas com a forma clínica virchowiana, sem tratamento, são consideradas os principais focos de transmissão da doença ${ }^{17}$. Portanto, a busca ativa de casos multibacilares em idosos do sexo masculino deve ser desenvolvida como importante estratégia para a prevenção de casos novos de hanseníase na comunidade.

Vale salientar que no nosso estudo a forma virchowiana foi mais frequente nos idosos do que nas demais faixas etárias, o que corrobora a hipótese da influência da imunocenessência. As diferentes manifestações clínicas da hanseníase estão relacionadas com a resposta imune à infecção pelo M. leprae, especialmente na forma clínica virchowiana, comprovadamente associada à baixa imunidade celular frente ao bacilo ${ }^{17}$. Nessa perspectiva, as taxas de detecção da hanseníase seriam maiores em idosos, independentemente da intensidade de exposição ao M. leprae, do diagnóstico tardio ou da força de transmissão da endemia 5 .

Evidenciou-se ainda que apenas 4,5\% dos idosos foram detectados pelo exame de contatos, e que para os demais grupos etários este modo de detecção variou de 14,2 a 6,2\%. Pedrosa et al. 18 verificaram que crianças diagnosticadas com hanseníase tiveram pelo menos um contato intradomiciliar positivo, das quais parte destes eram os avós, todos multibacilares. O contato com avós infectados foi considerado a fonte mais provável de infecção nessas crianças.

Para interromper a cadeia de transmissão, a vigilância de contatos é apontada como uma importante estratégia, pois garante o diagnóstico precoce e a prevenção de complicações relacionadas à doença. A vigilância ativa de contatos é estratégia eficaz para o controle da doença ${ }^{19}$. Tem como finalidade a descoberta de casos novos entre pessoas que convivem ou conviveram, de forma prolongada, com um caso de hanseníase. O objetivo é descobrir as fontes de infecção no domicilio ou fora dele 10.

Apesar dos diferentes níveis de endemicidade da hanseníase no Brasil 4,10, foram encontradas maiores taxas de detecção da doença nos idosos, quando comparadas às demais faixas etárias. Nobre et al. 5 e Dornels et al. 20 encontraram resultados semelhantes, para o Brasil e a Bahia, respectivamente. Esse perfil se deve ao longo período de incubação dos casos multibacilares, mais frequente nos idosos 21.

Segundo Irgens 21, em áreas com reduzida incidência da hanseníase esperam-se taxas de detecção baixas na faixa etária de 0-14 anos de idade e altas em idosos, que continuam a adoecer ainda que tenham sido infectados muitos anos antes da interrupção da transmissão. Por sua vez, altas taxas em menores de 15 anos indicam precocidade e intensidade da exposição ao bacilo e persistência de transmissão ativa da doença, padrão não esperado em locais onde há declínio da endemia 22. Entretanto, neste estudo, a taxa de detecção de casos novos foi maior nos idosos, inclusive nas UF com elevada detecção em menores de 15 anos 23, tais como os estados do Mato Grosso, Pará, Maranhão, Rondônia, Roraima, Pernambuco, Piauí e Acre, que se mantêm com parâmetro hiperendêmico 24.

O fato de haver maior taxa de detecção nos idosos também nos locais onde existe elevada transmissão em menores de 15 anos, aponta para a existência de outros fatores que podem contribuir para a maior carga da doença neste grupo etário. Uma das explicações pode ser a existência de fatores biológicos, como o declínio natural do sistema imunológico, que provoca aumento da susceptibilidade às doenças, redução da efetividade da vacinação e maior incidência de patologias associadas 25 .

Outro fato observado neste estudo foi que a taxa de GIF2 no diagnóstico dos casos novos de hanseníase também foi nitidamente maior nos idosos do que em outras faixas etárias, tanto para o Brasil como para todas as regiões e UF, corroborando o estudo anterior 4.

A ocorrência de incapacidades físicas em pessoas acometidas pela hanseníase significa diagnóstico tardio 2, que pode ser decorrente da falta de esclarecimento da população sobre os sinais e sintomas da doença, ao despreparo dos profissionais de saúde e ainda à evolução insidiosa da doença. De acordo com Henry et al. 26, pessoas que suspeitam ter hanseníase, mas temem a discriminação, têm dez vezes a chance de retardar o tempo de procura por um médico em relação aos que não temem. Aqueles que negligenciam seus sintomas e os que inicialmente recebem outro diagnóstico que não seja hanseníase, têm três vezes a chance de ser diagnosticados com incapacidades físicas 26.

Essas incapacidades podem ocasionar sequelas permanentes, pois decorrem de lesões dos nervos periféricos responsáveis pela sensação de dor, tato e calor, além do comprometimento de estruturas responsáveis pela visão. Isso torna o indivíduo susceptível a acidentes, queimaduras, feridas, infecções e amputações, além de promover danos sociais e psíquicos que interferem na qualidade de vida 27. Portanto, os presentes resultados apontam não apenas para a necessidade de tornar o diagnóstico 
mais precoce, mas também de oferecer condições adequadas para a reabilitação física desses idosos na rede de atenção à saúde do Sistema Único de Saúde (SUS) 4.

Dentre as limitações deste estudo, destacam-se aquelas inerentes ao uso de dados secundários do SINAN, que apresentam diferenças de cobertura e qualidade de dados entre as UFs, minimizadas pelo grande número de casos incluídos na pesquisa. Há também problemas relacionados a fatores operacionais ligados às ações de controle da endemia, como a insuficiência na busca ativa e diagnóstico de casos, que podem resultar na subnotificação, afetando a validade das taxas calculadas 23 . Essas limitações são apontadas por alguns autores que relatam problemas como a redução do diagnóstico de casos, em consequência de uma menor busca ativa, como fatores que podem interferir na endemicidade da doença 18 .

No entanto, esses fatores operacionais afetariam todos os grupos etários, especialmente o dos idosos que apresentaram as mais elevadas taxas de detecção, tornando os nossos achados ainda mais relevantes. Ressalta-se ainda que um estudo de avaliação do sistema de vigilância da hanseníase realizado nos estados da Amazônia Legal, no período de 2001-2007, revelou que o sistema foi útil, simples, com boa qualidade de dados e representativo para a descrição do perfil sociodemográfico e clínico dos casos 28.

Em conclusão, os achados revelam importantes diferenças no perfil epidemiológico e clínico da hanseníase nos idosos, em relação às demais faixas etárias. Todos os grupos apresentaram um perfil de baixa escolaridade e alta proporção de casos com raça/cor da pele negra, o que reforça a influência de fatores socioeconômicos no acometimento pela doença. Entre os idosos com hanseníase, predominaram multibacilares e baixa detecção por exame de contatos. Os idosos também exibiram as maiores taxas de detecção e de GIF2, o que ressalta a importância desta população na cadeia de transmissão da doença. Assim, evidencia-se a necessidade da adoção de estratégias específicas para esse grupo populacional que contemplem a heterogeneidade da situação da hanseníase no país.

Apesar de iniciativas já realizadas, como a publicação da Nota Informativa Conjunta no 01, de 10 de agosto de 2016, pelas Secretarias de Vigilância em Saúde e Assistência à Saúde, do Ministério da Saúde 29, que alerta sobre a necessidade do exame sistemático de hanseníase na população masculina e em idosos, é necessário monitorar a adesão dos serviços de saúde a estas recomendações, o fortalecimento da vigilância de contatos e o estreitamento da integração entre as áreas técnicas de hanseníase e de saúde do idoso nas três esferas de gestão do SUS. 


\section{Colaboradores}

M. C. N. Rocha contribuiu com a concepção e projeto do estudo, análise e interpretação dos dados e redação do artigo. M. L. Nobre e L. P. Garcia contribuíram com a análise e interpretação dos dados e revisão crítica relevante do conteúdo intelectual. Todos os autores aprovaram a versão final a ser publicada e são responsáveis por todos os aspectos do trabalho na garantia da exatidão e integridade de qualquer parte da obra.

\section{Informações adicionais}

ORCID: Margarida Cristiana Napoleão Rocha (0000-0002-2629-4532); Maurício Lisboa Nobre (0000-0003-4932-3137); Leila Posenato Garcia (0000-0003-1146-2641).

\section{Agradecimentos}

Aos revisores pela leitura e contribuições valiosas. À Dra. Vera Lúcia Gomes de Andrade pelo apoio e disponibilidade nas discussões sobre o tema.

\section{Referências}

1. World Health Organization. Global leprosy update, 2017: reducing the disease burden due to leprosy. Wkly Epidemiol Rec 2018; 93:44556.

2. Organização Mundial de Saúde. Estratégia Global para Hanseníase 2016-2020: acelerar rumo a um mundo sem hanseníase. https://apps.who.int/iris/bitstream/han dle/10665/254907/9789290225881-por.pdf? sequence $=8$ (acessado em 26/Fev/2019).

3. Rede Interagencial de Informação para a Saúde. Indicadores básicos para a saúde no Brasil: conceitos e aplicações. 2a Ed. Brasília: Organização Pan-Americana da Saúde; 2008.

4. Secretaria de Vigilância em Saúde, Ministério da Saúde. Hanseníase. Boletim Epidemiológico 2018; 49(4)

5. Nobre ML, Illarramendi X, Dupnik KM, Hacker MA, Nery JAC, Jerônimo SMB, et al. Multibacillary leprosy by population groups in Brazil: Lessons from an observational study. PLoS Negl Trop Dis 2017; 11:e0005364.

6. Lepra org. International day for older people. https://www.lepra.org.uk/News/internation al-day-for-older-people. (acessado em 19/ Fev/2019)

7. Diniz, LM, Maciel LB. Leprosy: clinical and epidemiological study in patients above 60 years in Espírito Santo State - Brazil. An Bras Dermatol 2018; 93:824-8.

8. Senado Federal. Estatuto da Igualdade Racial. Dispositivos constitucionais pertinentes: Lei no 12.288 , de 20 de julho de 2010. Índice temático. https://www2.senado.leg.br/bdsf/hand le/id/496308 (acessado em 03/Out/2019).

9. Instituto Brasileiro de Geografia e Estatística. Estatísticas de gênero. https://ww2.ibge.gov. $\mathrm{br} / \mathrm{apps} / \mathrm{snig} / \mathrm{v} 1 /$ notas_metodologicas.html (acessado em 07/Jan/2019).

10. Departamento de Vigilância das Doenças Transmissíveis, Secretaria de Vigilância em Saúde, Ministério da Saúde. Diretrizes para vigilância, atenção e eliminação da Hanseníase como problema de saúde pública: manual técnico-operacional. http://portalarquivos2. saude.gov.br/images/pdf/2016/fevereiro/04/ diretrizes-eliminacao-hanseniase-4fev16-web. pdf (acessado em 06/Nov/2018).

11. Nogueira PSF, Marques MB, Coutinho JFV, Maia JC, Silva MJ, Moura ERF. Factors associated with the functional capacity of older adults with leprosy. Rev Bras Enferm 2017; 70:711-8.

12. Gomes R, Nascimento EF, Araújo FC. Por que os homens buscam menos os serviços de saúde do que as mulheres? As explicações de homens com baixa escolaridade e homens com ensino superior. Cad Saúde Pública 2007; 23:565-74.

13. Silva DDB, Tavares CM, Gomes NMC, Cardoso AC, Arcêncio RA, Nogueira PSF. Leprosy in the elderly population of Alagoas. Rev Bras Geriatr Gerontol 2018; 21:553-61. 
14. Freitas LRS, Duarte EC, Garcia LP. Leprosy in Brazil and its association with characteristics of municipalities: ecological study, 2009-2011. Trop Med Int Health 2014; 19:1216-25.

15. Matos AMF, Coelho ACO, Araújo LPT, Alves MJM, Baquero OS, Duthie MS, et al. Assessing epidemiology of leprosy and socio-economic distribution of cases. Epidemiol Infect 2018; 146:1750-5.

16. Oliveira JSdS, Reis ALM, Margalho LP, Lopes GL, Silva ARd, Moraes NS, et al. Leprosy in elderly people and the profile of a retrospective cohort in an endemic region of the Brazilian Amazon. PLoS Negl Trop Dis 2019; 13:e0007709.

17. Talhari S, Andrade ARC, Ramos AMC, Talhari C, Braga B, Gonçalves HS. Manifestações cutâneas e diagnóstico diferencial. In: Talhari S, Penna GO, Gonçalves HS, Oliveira MLW, organizadores. Hanseníase. 5a Ed. Rio de Janeiro: Di Livros Editora; 2015. p. 17-43.

18. Pedrosa VL, Dias LC, Galban E, Leturiondo A, Palheta J Jr, Santos M, et al. Leprosy among schoolchildren in the Amazon region: A crosssectional study of active search and possible source of infection by contact tracing. PLoS Negl Trop Dis 2018; 12:e006261.

19. Hacker MA, Duppre NC, Nery JAC, Sales AM, Sarno EN. Characteristics of leprosy diagnosed through the surveillance of contacts: a comparison with index cases in Rio de Janeiro, 1987-2010. Mem Inst Oswaldo Cruz 2012; 107 Suppl 1:49-54.

20. Souza CDF, Fernandes TRMO, Matos TS, Ribeiro Filho JM, Almeida GKA, Lima JCB, et al. Grau de incapacidade física na população idosa afetada pela hanseníase no Estado da Bahia, Brasil. Acta Fisiátrica 2017; 24:27-32.

21. Irgens LM, Secular trends in leprosy: increase in age at onset associated with declining rates and long incubation periods. Int J Lepr Other Mycobact Dis 1985; 53:610-7.
22. Ferreira MAA, Osório MAR, Costa FO. Hanseníase na infância. In: Lyon S, Grossi MAF, organizadores. Hanseníase. Rio de Janeiro: Medbook; 2013. p. 67-9.

23. Freitas LRS, Duarte EC, Garcia LP. Trends of main indicators of leprosy in Brazilian municipalities with high risk of leprosy transmission, 2001-2012. BMC Infect Dis 2016; 16:472.

24. Barros PB, Freitas BHBM. Tendência da hanseníase em menores de 15 anos no Brasil, 20012016. Cad Saúde Pública 2018; 34:e00101817.

25. Chou JP, Effros RB. T cell replicative senescence in human aging. Curr Pharm Des 2013; 19:1680-98.

26. Henry M, GalAn N, Teasdale K, Prado R, Amar $\mathrm{H}$, Rays MS, et al. Factors contributing to the delay in diagnosis and continued transmission of leprosy in Brazil - an explorative, quantitative, questionnaire based study. PLoS Negl Trop Dis 2016; 10:e0004542.

27. Seshadri D, Khaitan BK, Khanna N, Sagar R. Dehabilitation in the era of elimination and rehabilitation: a study of 100 leprosy patients from a tertiary care hospital in India. Lepr Rev 2015; 86:62-74.

28. Freitas FTM, Sena Neto SA, Grossi MAF, Macário EM, Nóbrega AA. Evaluation of leprosy surveillance system in the Amazon region, Brazil, 2001-2007. Lepr Rev 2012; 86:5263.

29. Departamento de Vigilância das doenças transmissíveis, Secretaria de Vigilância em Saúde, Ministério da Saúde. Nota informativa conjunta no 01 de 2016. Nota informativa conjunta - alerta para o exame sistemático de hanseníase na população masculina e em idosos. http://portalsaude.saude.gov.br/images/ pdf/2016/setembro/06/Nota-InformativaConjunta-n---01--SAS-e-SVS--para-publica---o.pdf (acessado em 07/Jan/2019). 


\section{Abstract}

The article aimed to describe epidemiological indicators and characteristics of new cases of leprosy in elderly Brazilians in 2016-2018, compared to other age groups. A descriptive cross-sectional cohort study was conducted with data from the Information System on Diseases of Notification (SINAN). New leprosy cases were categorized by age groups: 60 or more, 40-59, 15-39, and 15 years of age. Pearson's chi-square test was used to verify differences between groups. A total of 81,205 new leprosy cases were reported in Brazil. Of these, 24.1\% were elderly, 37.7\% from 40-59 years, $31.9 \%$ from 15-39 years, and 6.3\% in individuals under 15 years of age. The elderly showed higher proportions $(p<0.001)$ of cases in males $(60.1 \%)$, with multibacillary classification (81.3\%), and with physical disability grade 2 (PDG2) (11.4\%) when compared to the other groups. However, the proportion of new cases detected in the elderly based on contact testing ( $4.9 \%)$ was the lowest among all the age brackets $(p<0.001)$. The mean detection rate and rate of new cases with PDG2 at diagnosis were higher among the elderly (25.1/100,000 and 28.6/million inhabitants, respectively) compared to other age groups in Brazil as a whole and in the regions and states. Important differences were seen in the epidemiological and clinical profile of leprosy in the elderly compared to other age brackets, especially higher proportions of multibacillary cases, new cases with PDG2, and low detection by contact testing. The findings highlighted the need for leprosy control in this age group, aimed at contributing to interruption of transmission of the disease.

Leprosy; Aged; Descriptive Epidemiology; Aged Groups

\section{Resumen}

El objetivo fue describir indicadores epidemiológicos y características de los nuevos casos de hanseniasis en ancianos en Brasil, durante el trienio 2016-2018, comparándolos con otros grupos etarios. Se realizó un estudio descriptivo de corte transversal con datos del Sistema de Información sobre Enfermedades de Notificación obligatoria (Sinan). Los nuevos casos de hanseniasis fueron categorizados por grupos etarios: 60 o más, 40-59, 15-39 y menores de 15 años. Se utilizó el test chi-cuadrado de Pearson para probar diferencias entre grupos. Se notificaron 81.205 casos nuevos de hanseniasis en Brasil. De estos, un 24,1\% fueron en ancianos, 37,7\% de 40-59 anos, 31,9\% de 15-39 años y 6,3\% en menores de 15 años. En los ancianos, se observaron proporciones mayores ( $p$ $<0,001)$ de casos del sexo masculino $(60,1 \%)$, con clasificación operacional multibacilar $(81,3 \%)$ y con grado 2 de incapacidad física (GIF2) (11,4\%), respecto a otros grupos. No obstante, la proporción de casos nuevos detectados en ancianos, por examen de contactos (4,9\%), fue la menor entre todas las franjas de edad $(p<0,001)$. Las tasas medias de detección y de casos nuevos con GIF2 en el diagnóstico fueron mayores entre ancianos (25,1/100.000 y 28,6/1 millón de habitantes, respectivamente), en comparación con los demás grupos etarios, respecto al país, regiones y Unidades de la Federación. Se observaron importantes diferencias en el perfil epidemiológico y clínico de la hanseniasis en ancianos, en relación con las demás franjas de edad, destacándose mayores proporciones de casos multibacilares, de casos nuevos con GIF2 y baja detección por examen de contactos. Se evidencia la necesidad del control de la hanseniasis en esa población, con el fin de contribuir a la interrupción de la transmisión de la enfermedad.

Lepra; Anciano; Epidemiología Descriptiva; Grupos de Edad
Recebido em 11/Mar/2019

Versão final 13/Nov/2019

Aprovado em 13/Fev/2020 